\title{
Correlación del Apiñamiento dental e Higiene Oral en escolares de Cuenca Ecuador
}

\author{
Correlation of dental crowding and Oral Hygiene in schoolchildren from \\ Cuenca Ecuador
}

Pablo Galarza Ulloa ${ }^{1 a}$, Miriam Verónica Lima Illescas $^{1 \mathrm{a}}$, Jaime Eduardo Pesántez Rodríguez ${ }^{1 \mathrm{a}}$, Santiago Daniel Serrano Piedra ${ }^{1 a}$

\section{RESUMEN}

Objetivo: El apiñamiento dental, sumado a una deficiente higiene bucal puede derivar en patologías gingivales y dentales, además de problemas estéticos y funcionales del sistema estomatognático. El objetivo de este estudio fue correlacionar el apiñamiento dental y la Higiene Oral en escolares de 12 años de edad del área urbana "El vecino" de la ciudad de Cuenca, Ecuador. Materiales y método Se realizó un estudio documental, descriptivo con un nivel relacional, retrospectivo, en base al macro estudio del Mapa Epidemiológico de Salud Bucal de Cuenca, del año 2016. Se utilizó toda la población, un total de 278 fichas clínicas de escolares de 12 años de edad. La prueba estadística que se utilizó fue el Coeficiente de Concordancia Tau-b Kendall con un nivel de seguridad del $95 \%(p<0,05)$. Resultados. La presencia de apiñamiento dental anterior fue el $41,7 \%$, el más frecuente fue el leve en el $60,3 \%$. Los niveles de Higiene Oral fueron bueno en el 60,4\%, seguido por el nivel regular con el 33,8\%. No existió relación entre el apiñamiento dental y el índice de Higiene oral $(p=0,794)$. Conclusiones. El apiñamiento dental no es indicador de mala higiene bucal en los participantes de esta área urbana. Los resultados positivos alientan a seguir trabajando con programas de educación de la salud bucal y campañas de prevención hacia la comunidad.

Palabras claves: Maloclusión; Índice de Higiene Oral; Niño; Ficha clínica. (Fuente: DeCS BIREME)

\section{ABSTRACT}

Objetive. Dental crowding added to poor oral hygiene can lead to gingival and dental pathologies and cause aesthetic and functional problems of the stomatognathic system. The objective of this study was correlate dental crowding and Oral Hygiene in 12 year old school students in the urban area "El vecino" of the city of Cuenca, Ecuador. Materials and Methods. documentary study was carried out, descriptive with a relational level, retrospective, based on the macro study of the Epidemiological Map of Oral Health of Cuenca, of the year 2016. The entire population was used, a total of 278 clinical records of 12 year old schoolchildren. The statistical test applied a Tau-b Kendall Match Coefficient with a safety level of $95 \%$ ( $p<0.05$ ). Results. The presence of previous dental crowding was $41.7 \%$, the most common was mild at $60.3 \%$. Oral Hygiene levels were good at $60.4 \%$, followed by regular level with $33.8 \%$. There was no link between dental crowding and the Oral Hygiene Index ( $\mathrm{p}-0.794)$. Conclusions. Dental crowding is not an indicator of poor oral hygiene in participants in this urban area. Positive results encourage further work with oral health education programs and community prevention campaigns.

Key words:. Malocclusion; Oral Hygiene index; Child; Clinical record (Source: MeSH NLM)

Recibido: 20 de marzo de 2020

Facultad de Odontología. Universidad Católica de Cuenca. Cuenca-Ecuador.

a Docente

Correspondencia:

Pablo Galarza Ulloa

Correo electrónico: ulloasan115@gmail.com
Publicado: 06 de julio de 2020

Este es un artículo Open Access distribuido bajo la licencia Creative Commons Atribución-No Comercial- Compartir Igual 4.0

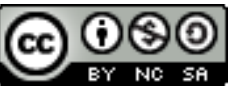

Citar como: Galarza Ulloa P, Lima Illescas M, Pesántez Rodríguez J, Serrano Piedra S. Correlación del Apiñamiento dental e Higiene Oral en escolares de Cuenca Ecuador. KIRU. 2020; 17(3): 129-134.https://doi.org/10.24265/kiru.2020.v17n3. 


\section{INTRODUCCIÓN}

La maloclusión es una alteración de la posición de los dientes y de los arcos basales, que causa deterioro en la estética en casos ligeros y alteraciones anatómico funcional en los más graves (1). Su etiología es por la compleja interacción de factores genéticos y del medio ambiente $^{(2,3)}$.

La maloclusión ocupa el tercer lugar de las enfermedades más prevalentes de la cavidad oral según la Organización Mundial de la Salud (OMS) $(1,2,4)$ y el apiñamiento dental en el sector anterior es la anomalía más percibido por los individuos. La frecuencia de apiñamiento en niños varía desde el $26,6 \%{ }^{(5)}$ hasta el $46,8 \%{ }^{(1)}$ mostrando predominancia el leve, este último reportado en la ciudad de la presenta investigación (6). Una característica importante es la estética y la funcionalidad que cumplen las piezas dentales, por lo que el apiñamiento constituye el motivo de consulta más común en la atención dental ${ }^{(7-10)}$.

Alrededor del $65 \%$ de los escolares, exhiben una deficiencia en la salud bucal, ligado directamente con la higiene oral, este es uno de los factores imprescindibles para poseer una óptima salud buco dental (5). Uno de los parámetros más utilizados para diagnosticar la presencia de placa dental blanda y dura es el Índice de Higiene Oral Simplificado de Greene y Vermillion (IHOS) ${ }^{(11)}$. La frecuencia de HIOS reportado fue bueno en escolares perteneciente a áreas urbanas ${ }^{(11)}$ y rurales ${ }^{(5,12)}$ de la ciudad del presente estudio.

La severidad del apiñamiento puede relacionarse con una deficiente higiene bucal, ya que puede desencadenar en el acúmulo de residuos alimenticios y consecuentemente producir otra clase de problemas como enfermedad en las encías, periodonto, caries dental ${ }^{(4,7,8)}$, movilidad dentaria e incluso se puede llegar a la pérdida de la pieza dental considerados como problemas de salud pública ${ }^{(1,13,14)}$. Estas afecciones del sistema estomatognático provocan afecciones funcionales, emocionales y sociales en el individuo ${ }^{(5,15,16,17)}$.

Partiendo del supuesto que la presencia de apiñamiento influye en una higiene bucal defectuosa, con deterioro de la salud bucal, el propósito del presente trabajo fue correlacionar el apiñamiento y la higiene oral en escolares de 12 años de edad de la parroquia urbana El Vecino de la ciudad de Cuenca- Ecuador.

\section{MATERIALES Y MÉTODOS}

Se realizó un estudio documental, descriptivo con nivel relacional, retrospectivo, en base al macro estudio del Mapa Epidemiológico de Salud Bucal de la parroquia El Vecino, esta información reposa en la Facultad de Odontología de la Universidad Católica de Cuenca. Se utilizó toda la población, 278 fichas que cumplieron con los criterios de inclusión tales como fichas epidemiológicas de escolares de 12 años de edad que pertenecen a esta área urbana, que proporcione la información acerca de las variables de estudio y se excluyó fichas que no presentan datos completos 0 ilegibles. Los datos extraídos fueron número de registro, edad, sexo, tipo de gestión educativa, apiñamiento dental anterior y el Índice de Higiene oral.

Participaron cinco instituciones educativas, (Juan Montalvo, Luis Roberto Bravo, Mary Coryle, Republica de Chile, Zoila Esperanza P) pertenecientes a la parroquia El Vecino, este sector está ubicado en límite norte del área urbano de la ciudad de Cuenca.

La variable independiente fue el apiñamiento dental anterior, que se determinó mediante su severidad como: ausencia de apiñamiento $(0 \mathrm{~mm}$.), apiñamiento leve (1-3mm.), apiñamiento moderado $(>3-5 \mathrm{~mm})$ y apiñamiento severo $(>5 \mathrm{~mm})^{(1,4)}$, mientras que la variable dependiente fue el IHOS, y se estableció los niveles de higiene oral como: excelente $(0,0)$, bueno $(1,2)$, regular $(1,3-3,0)$ y malo $(3,1-6,0)^{(5,11,12)}$. Además, como covariables fue el sexo y tipo de gestión educativa.

Los resultados se expresaron en tablas de frecuencia absoluta y porcentual y se empleó el Coeficiente de concordancia Tau-b de Kendall, para evaluar el grado de relación de las variables con escala ordinal y una seguridad del $95 \%$ $(p<0,05)$. Los datos obtenidos se procesaron empleando el programa estadístico Statistical Package for Social Sciences (SPSS) versión 25, la edición de tablas y gráficos se elaboró en Microsoft Excel 2013, Windows 10.

La presente investigación no implicó ningún conflicto bioético, ya que se ejecutó sobre datos retrospectivos los mismo que reposan en la Facultad de Odontología, además se guardará la respectiva confidencialidad sobre la información proporcionada. 


\section{RESULTADOS}

El $61,5 \% \quad(n=171)$ de los escolares fueron mujeres y el $38,5 \%(n=107)$ fueron hombres con un total de 278 estudiantes. El tipo de gestión académica fueron en su totalidad escuelas fiscales. La presencia de apiñamiento dental fue el $41,7 \%$; mientras que en la severidad la más frecuente fue el apiñamiento leve en el 60,3\%. En los niveles del Índice de Higiene Oral el más frecuente fue un nivel bueno en el $60,4 \%$ y le sigue el regular con el $33,8 \%$ (tabla 1 ).

Tabla 1. Distribución de las Variables

\begin{tabular}{lcc}
\multicolumn{1}{c}{ VARIABLES } & $\mathbf{n}$ & $\%$ \\
PRESENCIA DE APIÑAMIENTO & & \\
No & 162 & 58,3 \\
Sí & 116 & 41,7 \\
Total & 278 & 100 \\
SEVERIDAD DE APIÑAMIENTO & & \\
Leve & 70 & 60,3 \\
Moderada & 29 & 25 \\
Severo & 17 & 14,7 \\
Total & 116 & 100 \\
NIVELES DE IHO & & \\
Malo & 1 & 0,4 \\
Regular & 94 & 33,8 \\
Bueno & 168 & 60,4 \\
Excelente & 15 & 5,4 \\
Total & 278 & 100 \\
\hline ración propia, tomada del Mapa Epidemiológico de Salud Bucal de Cuenca, 2016
\end{tabular}

En la tabla 2 se observa que el $41,7 \%$ de los escolares presentaron apiñamiento dental, el $42,1 \%$ fueron hombres y el $41,5 \%$ fueron mujeres.
En cuanto a la severidad de apiñamiento, el nivel más frecuente fue el leve en ambos sexos, con el $60,6 \%$ en mujeres y $60,3 \%$ en hombres (tabla 3 ).

Tabla 2. Presencia de apiñamiento dentario de acuerdo con el sexo

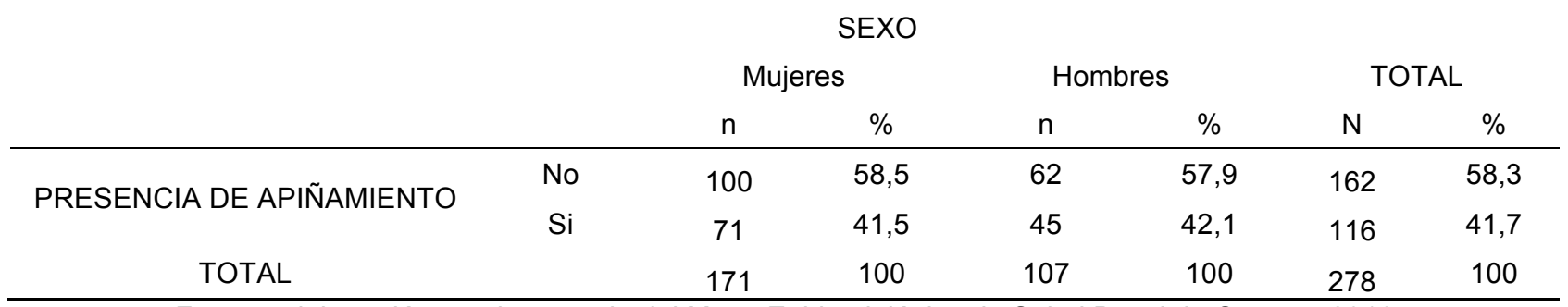

Fuente: elaboración propia, tomada del Mapa Epidemiológico de Salud Bucal de Cuenca, 2016 
Tabla 3. Severidad de Apiñamiento de acuerdo con el sexo

\begin{tabular}{lccccccc} 
& & \multicolumn{2}{c}{ SEXO } & \multicolumn{2}{c}{ Hombres } & \multicolumn{2}{c}{ TOTAL } \\
\cline { 2 - 8 } SEVERIDAD DE APIÑAMIENTO & & $\mathrm{n}$ & $\%$ & $\mathrm{n}$ & $\%$ & $\mathrm{~N}$ & $\%$ \\
\cline { 2 - 8 } & Leve & 43 & 60,6 & 27 & 60 & 70 & 60,3 \\
& Moderado & 18 & 25,4 & 11 & 24,4 & 29 & 25 \\
Total & 10 & 14 & 7 & 15,6 & 17 & 14,7 \\
& Severo & 71 & 100 & 45 & 100 & 116 & 100 \\
\hline
\end{tabular}

Fuente: elaboración propia, tomada del Mapa Epidemiológico de Salud Bucal de Cuenca, 2016

En cuanto a los niveles de Higiene Oral, se observó que el mayor número de escolares presentó buena higiene oral con el $69,2 \%$ en los hombres, seguido de una higiene regular con el $29 \%$; mientras que el $55,6 \%$ corresponde a una higiene buena en las mujeres. Para el nivel de Higiene Oral malo no existieron casos significativos, entre ambos sexos, la cual se presenta en la tabla 4.

Tabla 4. Niveles de Higiene oral según el sexo

\begin{tabular}{|c|c|c|c|c|c|c|c|}
\hline & & \multicolumn{4}{|c|}{ SEXO } & \multirow{2}{*}{\multicolumn{2}{|c|}{ TOTAL }} \\
\hline & & \multicolumn{2}{|c|}{ Mujeres } & \multicolumn{2}{|c|}{ Hombres } & & \\
\hline & & $\mathrm{n}$ & $\%$ & $n$ & $\%$ & $\mathrm{~N}$ & $\%$ \\
\hline & Malo & 1 & 1 & 0 & 0 & 1 & 0,4 \\
\hline \multirow[t]{3}{*}{ NIVELES DE IHO } & Regular & 63 & 36,8 & 31 & 29 & 94 & 33,8 \\
\hline & Bueno & 95 & 55,6 & 74 & 69,2 & 169 & 60,8 \\
\hline & Excelente & 12 & 7 & 2 & 1,9 & 14 & 5 \\
\hline Total & & 171 & 100 & 107 & 100 & 278 & 100 \\
\hline
\end{tabular}

Fuente: elaboración propia, tomada del Mapa Epidemiológico de Salud Bucal de Cuenca, 2016

La prueba estadística del coeficiente de correlación de Kendall mostró ausencia de significación estadística al obtener el valor de $p$ mayor al $5 \%$ $(p=0,794)$, y su grado de asociación fue negativo e inverso $(-0,014)$ por lo que no existe relación entre el apiñamiento dentario y el IHOS, el detalle se observa en el gráfico 1. No se encontró relación de estas variables de acuerdo al sexo.

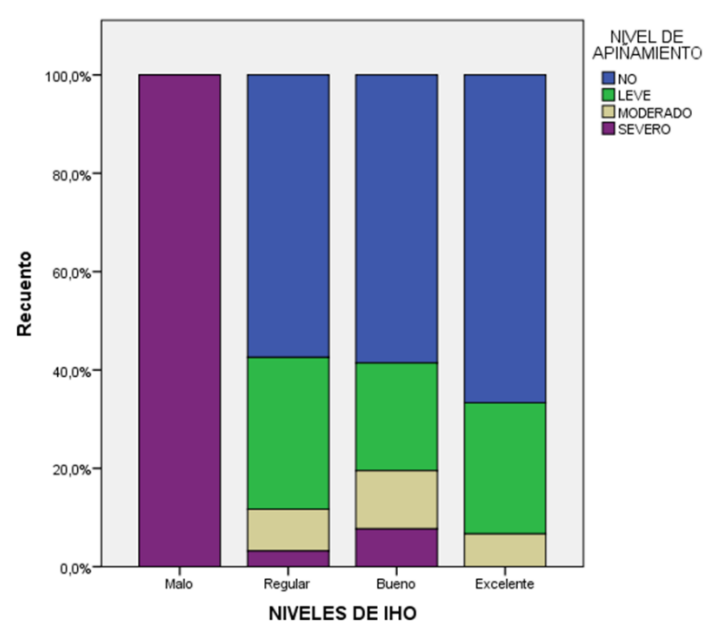

Gráfico 1. Relación de IHOS y Nivel de Apiñamiento

Coeficiente de correlación Tau-b Kendall = -0,014

Valor de $p=0,794$ 


\section{DISCUSIÓN}

El presente estudió se efectuó en instituciones educativas que pertenecen a la parroquia urbana "El Vecino", de la ciudad de Cuenca, la cual está conformada por 279 fichas clínicas, en niños de 12 años de edad, dando como resultado que el $38,5 \%$ fueron hombres, y el $61,5 \%$ mujeres. Porcentajes que tienen similitud con los resultados obtenidos por lbarra y cols. ${ }^{(15)}$, realizado en la misma parroquia en el año 2017, puesto que en el sexo masculino tuvo un porcentaje del $40 \%$, mientras que en el sexo femenino fue de $60 \%$, realizado en escolares de la misma edad.

Lima ${ }^{(1)}$ reportó un apiñamiento anterior del 46,8 $\%$; Salinas y cols. ${ }^{(18)}$, encontró un apiñamiento dental en el $36,2 \%$ en los hombres y el $30,5 \%$ en las mujeres. Similar fue los resultados de la presente investigación con un apiñamiento dental anterior del $41,7 \%$, con predominancia en los hombres en el $42,1 \%$ mientras que en las mujeres fue $41,5 \%$.

El apiñamiento dental de acuerdo a la severidad fue el $60,3 \%$ como leve, seguido con el $25 \%$ que corresponde el apiñamiento moderado, y únicamente el 14,7\% fue severo, por otro lado los resultados de García y cols. ${ }^{(19)}$, determinó con supremacía el apiñamiento leve en el 33,2\%, seguido del moderado con el $27,1 \%$, y finalmente el severo con el 3,7\%.

De acuerdo a los niveles del índice de Higiene Oral fue predominante el Índice bueno en el $69,2 \%$ en los participantes de sexo masculino, mientras que en las mujeres fue el 55,6\%; análogo a los resultados reportados por Vega ${ }^{(12)}$, en el año 2016 en la parroquia rural Sayausí de la ciudad de Cuenca, manifestó que el sexo masculino prevaleció con un IHOS bueno en el $65 \%$, a comparación del sexo femenino con el $64 \%$.

En cuanto a la correlación de apiñamiento dental e Índice de Higiene Oral, se determinó que no existe asociación, es importante recalcar que no existen estudios con asociación directa de estas variables a nivel local y nacional, sin embargo, Sánchez y cols. ${ }^{(6)}$, en una investigación en la ciudad de México encontró una relación significativa entre el apiñamiento y el IHOS en los escolares participantes. Por otro lado, García ${ }^{(19)}$ determinó relación significativa entre apiñamiento anterior leve e inflamación gingival leve.

En el presente estudio se determinó que el Apiñamiento dental no es indicador de mala higiene bucal por la ausencia de correlación de estas variables, por lo que los participantes de esta área urbana, presentaron una buena higiene oral. Los resultados positivos alientan a seguir trabajando con mayor eficacia mediante programas de educación de salud bucal y campañas de prevención hacia la comunidad.

Contribución de autoría: PGU y MLI diseño del estudio, análisis e interpretación de los datos. PGU, JPE y SSP adquisición de los datos. La revisión crítica fue realizada por todos los autores.

Fuentes de financiamiento: autofinanciada.

Declaración de conflicto de Interés: Los autores declaran la no existencia de conflicto de interés.

\section{REFERENCIAS}

1. Lima $M$, Soto $L$, Peñafiel V, Soto P. Maloclusión dental en estudiantes de 4 a 15 años, en Cuenca, Ecuador. Invest Medicoquir. 2018 [citado 14 de Septiembre 2020]; 10 (2): [aprox. 15 p.] Disponible en: https://www.medigraphic.com/cgi$\mathrm{bin} / \mathrm{new} /$ resumen.cgi? IDARTICULO=85518

2. Ospina D, Herrera $Y$, Betancur J, Agudelo $H$, Posada A. Higiene bucal en la población de San Francisco, Colombia, y sus factores relacionados. Rev Nac Odontol. 2016 [citado 23 de Junio 2020]; 12 (22):23-30 Disponible en: https://revistas.ucc.edu.co/index.php/od/article/vi ew/1203/1402

3. Cabrera C, Arancet M, Martínez D, Cueto A, Espinoza S. Salud Oral en Población Escolar Urbana y Rural. Int. J. Odontostomat. 2015 [citado 23 de Junio 2020]; 9(3):341-348 Disponible en: https://scielo.conicyt.cl/pdf/ijodontos/v9n3/art01.p df

4. Fajardo J, González L. Prevalencia de maloclusiones dentales en escolares de 12 años en Monay - Cuenca 2016. Revista OACTIVA UC Cuenca. 2016 [citado 25 de Junio 2020]; 1 (2): 23-28 Disponible en: https://doi.org/10.31984/oactiva.v1i2.133

5. Jáuregui J, Vásquez A, Sacoto $F$. Índice de Higiene Oral en los Escolares de 12 años de la Parroquia Checa en el Cantón Cuenca, Provincia del Azuay - Ecuador, 2016. Odontoestomatología. 2019 [citado 23 de Junio 2020]; 21 (34): [aprox. 7 p.] Disponible en: http://www.scielo.edu.uy/pdf/ode/v21n34/16889339-ode-21-34-27.pdf

6. Sánchez L, Sáenz L, Alfaro P, Osorno C. Comportamiento del apiñamiento, gingivitis, higiene oral, caries, flujo salival y bacterias en escolares de 8 y 10 años. Rev ADM. 2013 [citado 14 de Septiembre 2020]; 70(2):91-97. Disponible en: https://www.academia.edu/17799560/Comporta miento del api\%C3\%B1amiento gingivitis higie ne_oral_caries_flujo_salival_y_bacterias_en_esc olares_de_8_y_10_a\%C3\%B10s 
7. Díaz H, Ochoa B, Paz L, Casanova K, Coca Y. Prevalencia de maloclusiones en niños de la escuela Carlos Cuquejo del municipio Puerto Padre, Las Tunas. Medisur. 2015 [citado 23 de Junio 2020]; 13(4): [aprox. 7p.] Disponible en: http://scielo.sld.cu/pdf/ms/v13n4/ms05413.pdf

8. Carmo M, Freire M, Morales L. Socioeconomic inequalities and changes in oral health behaviors among Brazilian adolescents from 2009 to 2012. Rev Saúde Pública. 2015 [citado 23 de Junio 2020]; 49:50 Disponible en: 8910.2015049005562

9. Castillo A, Mattos M, Castillo R, Mendoza C. Maloclusiones en niños y adolescentes de caseríos y comunidades nativas de la Amazonía de Ucayali, Perú. Rev Peru Med Exp Salud Pública. 2011 [citado 23 de Junio 2020]; 28(1): 87-91 Disponible en: http://www.scielo.org.pe/pdf/rins/v28n1/a14v28 n1.pdf

10. Santiesteban F, Gutiérrez M, Gutiérrez J. Severidad de apiñamiento relacionado con la masa dentaria. Revista Mexicana de Ortodoncia. 2016 [citado 23 de Junio 2020]; 4(3): 165-168 Disponible en: https://www.medigraphic.com/pdfs/ortodoncia/ mo-2016/mo163e.pdf

11. Rodríguez C, Calle M, Cabrera G, Encalada L. Índice de higiene oral simplificado en los escolares de 6 años de la parroquia Sucre, Cuenca, Azuay - Ecuador en el 2016. Revista Salud \& Vida Sipanense. 2018 [citado 25 de Junio 2020]; 5(1):37 -46 Disponible en: http://revistas.uss.edu.pe/index.php/SVS/articl e/view/927/787

12. Vega D. Índice de higiene oral en niños de 6 años en ecuador 2016. Revista OACTIVA UC Cuenca. 2016 [citado 23 de Junio 2020]; 1 (2): 39-44 Disponible en: https://doi.org/10.31984/oactiva.v1i2.144

13. Villagrana M, Becerra J, Rivas R, Aguilar S. Componentes de la maloclusión clase II esquelética en niños mexicanos de 8 a 12 años. Rev. Tamé. 2018 [citado 23 de Junio 2020]; 7 (19):708-711 Disponible en: https://www.medigraphic.com/pdfs/tame/tam2018/tam1819b.pdf
14. Suárez L, Castillo $R$, Brito $R$, Santana A, Vázquez $\mathrm{Y}$. Oclusión dentaria en pacientes con maloclusiones generales: asociación con el estado funcional del sistema estomatognático. Medicent Electrón. 2018 [citado 23 de Junio 2020]; 22(1) Disponible en: http://scielo.sld.cu/pdf/mdc/v22n1/mdc07118.p df

15. Ibarra K, Calle M, López E, Heredia D. Índice de higiene oral comunitario en escolares de 12 años. Rev. Evid. Odontol. Clinic. 2017 [citado 23 de Junio 2020]; 3 (2) Disponible en: http://dx.doi.org/10.35306/eoc.v3i2.495

16. Jiménez $A$, Cárdenas $C$. Asociación entre el índice de higiene oral y prevalencia de caries de infancia temprana en preescolares de 3-5 años de la institución educativa San Gabriel, Villa María del triunfo, 2017. Rev Cient Odontol (Lima). 2018 [citado 23 de Junio 2020]; 6 (2): 177-185 Disponible en: https://doi.org/10.21142/2523-2754-06022018-177-185

17. Lao, W, Araya H, Mena D. Prevalencia de apiñamiento dental en la población costarricense que consulta los servicios de odontología de la CCSS, 2017. Odontología Vital. 2019 [citado 23 de Junio 2020]; 30:39-44 Disponible en: https://www.scielo.sa.cr/pdf/odov/n30/16590775-odov-30-39.pdf

18. Salinas L, Urgiles $C$, Jiménez $M$. Maloclusiones dentales en escolares de 12 años en la parroquia el Sagrario - Cuenca 2016. Rev. Salud \& Vida Sipanense. 2017 [citado 23 de Junio 2020];4(2):58-66. Disponible en: http://revistas.uss.edu.pe/index.php/SVS/articl e/view/703/615

19. García V. Zhunio K. Prevalencia de apiñamiento dentario anterior y su relación con la gingivitis, en adolescentes de 12 a 15 años de la unidad educativa Tres de noviembre, parroquia Bellavista, Cuenca - 2015. Revista OACTIVA UC Cuenca. 2018 [citado 23 de Junio 2020]; 3(2): [aprox. 6 p.] Disponible en: https://oactiva.ucacue.edu.ec/index.php/oactiv a/article/view/216

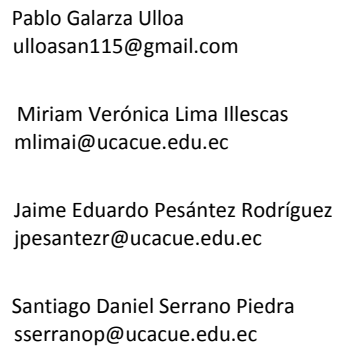

\footnotetext{
https://orcid.org/0000-0002-6562-1866

(b) https://orcid.org/0000-0001-6844-3826.

https://orcid.org/0000-0002-7431-3822

https://orcid.org/0000-0001-7899-6248
} 